\title{
ANÁLISE DA FUNÇÃO RESPIRATÓRIA NA DOENÇA DE PARKINSON
}

\author{
Sônia R.X. Cardoso', João S. Pereira²
}

\begin{abstract}
RESUMO - Avaliou-se a função respiratória de 40 parkinsonianos (P), entre 50 e 80 anos, nos estágio I a III da Escala de Hoehn e Yahr e de 40 não parkinsonianos (NP), com características semelhantes. A amplitude torácica de $1,8 \pm 0,8 \mathrm{~cm}$ nos $P$ foi menor que $4,3 \pm 1,0 \mathrm{~cm}$ nos NP $(p=0,00001)$, assim como os percentuais das capacidades vital e vital forçada de $66,8 \pm 20,3 \%$ e $69,6 \pm 22,2 \%$ nos P e de $82,3 \pm 15,7 \%$ e $82,7 \pm 16,6 \%$ nos NP $(p=0,00001$ e $p=0,0023)$. Apresentaram-se equivalentes as pressões inspiratória e expiratória máximas, de $33,5 \pm 12,7 \mathrm{cmH}_{2} \mathrm{O}$ e $36,3 \pm 17,8 \mathrm{cmH}_{2} \mathrm{O}$ nos $\mathrm{P}$ e de $37,0 \pm 12,2 \mathrm{cmH}_{2} \mathrm{O}$ e 43,1 $\pm 16,6 \mathrm{cmH}_{2} \mathrm{O}$ nos NP $(\mathrm{p}=0,1753$ e 0,0398 ), o volume do $1^{\circ}$ segundo da curva expiratória forçada de $71,3 \pm 25,6 \%$ nos $P$ e $80,6 \pm 23,6 \%$ nos NP $(p=0,0899)$ e o percentual da capacidade vital forçada expirada em um segundo, de $104,5 \pm 19,9 \%$ nos $P$ e $97,4 \pm 22,8 \%$ nos NP $(p=0,1234)$. Os parkinsonianos evidenciaram restrição respiratória e diminuição de amplitude torácica, sem alteração da força muscular respiratória.
\end{abstract}

PALAVRAS-CHAVE: função pulmonar, doença de Parkinson, restrição respiratória.

\begin{abstract}
Analysis of breathing function in Parkinson's disease
ABSTRACT - We studied 40 parkinsonian patients (P), mean age 50-80 years, with Hoehn and Yahr stages I-III and 40 no parkinsonian patients (NP), with similar characteristics. The results of the thoracic amplitude was $1,8 \pm 0,8 \mathrm{~cm}$ of $P$ that showed a reduction to $4,3 \pm 1,0 \mathrm{~cm}$ of NP $(p=0,00001)$, the vital capacity and forced vital capacity, $66,8 \pm 20,3 \%$ and $69,6 \pm 22,2 \%$ of $P$ was decreased that $82,3 \pm 15,7 \%$ and $82,7 \pm 16,6 \%$ of NP $(p=0,00001$ and $p=0,0023)$. There was not difference among the maximal inspiratory and expiratory mouth pressures, $33,5 \pm 12,7 \mathrm{cmH}_{2} \mathrm{O}$ and $36,3 \pm 17,8 \mathrm{cmH}_{2} \mathrm{O}$ of $\mathrm{P}$ and $37,0 \pm 12,2 \mathrm{cmH}_{2} \mathrm{O}$ and $43,1 \pm 16,6 \mathrm{cmH}_{2} \mathrm{O}$ of NP $(p=0,1753$ and $p=0,0398)$, the forced expiratory volume in 1 second, $71,3 \pm 25,6 \%$ of $P$ and $80,6 \pm 23,6 \%$ of NP $(p=0,0899)$, and the forced expiratory volume in 1 second/ forced vital capacity, $104,5 \pm 19,9 \%$ of $P$ and $97,4 \pm 22,8 \%$ of NP $(p=0,1234)$. The parkinsonian patients present restrictive pulmonary dysfunction, low chest wall compliance and the respiratory muscle strenght do not decreased.
\end{abstract}

KEY WORDS: breathing function, Parkinson's disease, restrictive pulmonary dysfunction.

Os estudos sobre as alterações da função respiratória na doença de Parkinson (DP) vêm merecendo destaque ao longo do tempo, embora não haja unanimidade quanto aos fatores de comprometimento nem quanto à limitação funcional imposta aos pacientes. Comprovou-se a presença de alteração da respiração nos portadores da DP, por diminuição da amplitude do tórax e dos volumes pulmonares ${ }^{1}$. A complacência pulmonar diminui pela limitação na extensão de tronco e da amplitude articular do tórax e da coluna vertebral, secundária a artrose e outras alterações torácicas como a cifoescoliose $^{2,3}$ ou fibrose pleural ${ }^{4}$. Portanto, a amplitude torácica diminuída em decorrência da postura em flexão do tronco ${ }^{5}$ e a degeneração ósteo-articular, alteram o eixo da coluna vertebral, o que repercute na inspiração e na expiração ${ }^{6}$. Com o envelhecimento, o sistema respiratório apresenta alterações estruturais, perda de elasticidade, dilatação alveolar, diminuição do estímulo neural para os músculos respiratórios e alterações de volumes, capacidades e fluxos respiratórios ${ }^{7}$.

A reabilitação dos portadores da DP tem sido direcionada especialmente para as limitações motoras, fazendo-se necessária avaliação funcional respiratória a fim de identificar as disfunções e estabelecer programa de tratamento direcionado.

\section{MÉTODO}

Foram selecionados, aleatoriamente, 40 indivíduos parkinsonianos (P) entre 50 e 80 anos, sendo 21 deles do

Estudo realizado no Ambulatório de Distúrbios do Movimento do Serviço de Neurologia do Hospital Universitário Pedro Ernesto da Universidade do Estado do Rio de Janeiro (UERJ) Rio de Janeiro RJ, Brasil: ${ }^{1}$ Mestre em Ciência da Motricidade Humana pela Universidade Castelo Branco (UCB-RJ), Professora Adjunta de Fisioterapia da Faculdade de Reabilitação da Associação de Solidariedade à Criança Excepcional (FRASCE-RJ); ${ }^{2}$ Doutor em Medicina pela Escola Paulista de Medicina (EPM-SP), Professor Adjunto da FCM-UERJ, Professor do PROCIMH / UCB-RJ.

Recebido 28 Maio 2001, recebido na forma final 25 Setembro 2001. Aceito 9 Outubro 2001. 
sexo masculino e 19 do feminino, em uso regular de Ldopa, associada ou não a outra medicação antiparkinsoniana, classificados na escala de Hoehn-Yahr ( $\mathrm{H}-\mathrm{Y})$ nos estágios de I a III, em acompanhamento no Ambulatório de Distúrbios do Movimento do Serviço de Neurologia do Hospital Universitário Pedro Ernesto/ Universidade do Estado do Rio de Janeiro (HUPE/UERJ). O outro grupo, que serviu como controle, era constituído de 40 indivíduos não parkinsonianos (NP), com características semelhantes quanto à idade, ao sexo e às atividades funcionais desempenhadas.

Após esclarecimento detalhado sobre a pesquisa e a formalização para a participação com carta de consentimento, foram agendadas e realizadas, pela manhã, as avaliações da função respiratória, com todos participantes medicados. Realizou-se a anamnese, demonstração, treinamento e aferição das medidas de: estatura $(\mathrm{em} \mathrm{cm})$, com antropômetro, pela distância do vértex ao plano, estando em apnéia inspiratória, posição anatômica e apoio posterior; amplitude torácica $(\mathrm{em} \mathrm{cm})$, com trena ao redor do tórax no nível do apêndice xifóide, em expiração normal, na inspiração e na expiração máximas; força inspiratória e expiratória máximas (em $\mathrm{cm}$ de água), com manovacuômetro, através de bocal, ocluindo-se o sistema ao final da inspiração e expiração máximas; expansibilidade pulmonar e permeabilidade de vias aéreas (em percentual de volumes, capacidades e fluxos teóricos), com espirômetro, através de bocal, da inspiração até a expiração máximas, tanto lenta quanto rapidamente. Os resultados foram enquadrados em: normal (N) e com distúrbios, sendo estes obstrutivo leve (OL), obstrutivo moderado $(\mathrm{OM})$, restritivo leve (RL), restritivo leve associado a obstrutivo leve (RLOL), restritivo leve associado a obstrutivo moderado (RLOM), restritivo moderado (RM) e restritivo severo (RS).

Foram observadas as considerações básicas no tratamento estatístico, com nível de significância de $p \leq 0,05$.
Foram utilizados: a estatística descritiva, caracterizando o universo amostral pesquisado; a estatística de inferência ANOVA one way e o teste de Tukey, para comparação intergrupos; o teste não paramétrico do qui-quadrado, para as variáveis de natureza discreta; o teste de correlação de Pearson, selecionando as relações funcionais de proporcionalidade.

\section{RESULTADOS}

A amostra constituída de 80 indivíduos, sendo 40 P e 40 NP, apresentava em cada grupo 21 indivíduos do sexo masculino e 19 do feminino, com média de idade $65,5 \pm 9,3$ anos, sendo determinadas três faixas etárias: de 50 a 59 anos, de 60 a 69 anos e de 70 a 80 anos (Tabela 1).

Foram medidas :a amplitude torácica inspiratória, determinada pela perimetria inspiratória (PerimIF), em $0,9 \pm 0,5 \mathrm{~cm}$ nos P e 2,2 $20,6 \mathrm{~cm}$ nos NP; a expiratória, determinada pela perimetria expiratória (PerimEF), em $1,0 \pm 0,4 \mathrm{~cm}$ nos $P$ e $2,1 \pm 0,5 \mathrm{~cm}$ nos NP e a total (AmplTOT), resultante do somatório da PerimIF com a PerimEF, em $1,8 \pm 0,8 \mathrm{~cm}$ nos $P$ e $4,3 \pm 1,0 \mathrm{~cm}$ nos NP. Todas evidenciaram diferença entre as médias dos grupos $\mathrm{P}$ e NP, sendo significativamente menores $(p=0,00001)$ nos grupos $P$ (Fig 1$)$.

A medida da força muscular inspiratória (Pimax) foi de $33,5 \pm 12,7 \mathrm{cmH}_{2} \mathrm{O}$ nos $\mathrm{P}$ e $37,0 \pm 12,2 \mathrm{cmH}_{2} \mathrm{O}$ nos NP e a expiratória (Pemax), de $36,3 \pm 17,8 \mathrm{cmH}_{2} \mathrm{O}$ nos $\mathrm{P}$ e $43,1 \pm 16,6 \mathrm{cmH}_{2} \mathrm{O}$ nos NP, não evidenciando diferenças entre as médias dos grupos P e NP (Fig 2).

A medida da capacidade vital em relação aos valores teóricos (\%CV), de $66,8 \pm 20,3 \%$ nos P e 82,3 $\pm 15,7 \%$ nos NP evidenciou diferença entre as médias dos grupos, sendo significativamente menor $(p=0,00001)$ nos P. A capacidade vital forçada em

Tabela 1. Características da amostra: grupo, sexo e faixa etária.

\begin{tabular}{|c|c|c|c|c|c|c|}
\hline \multicolumn{7}{|l|}{ Feminino } \\
\hline Grupo & \multicolumn{2}{|c|}{ Não parkinsoniano } & \multicolumn{2}{|c|}{ Parkinsoniano } & \multicolumn{2}{|c|}{ Geral } \\
\hline Faixa Etária & Absoluto & Relativo & Absoluto & Relativo & Absoluto & Relativo \\
\hline de 50 a 59 anos & 5 & $13,2 \%$ & 5 & $13,2 \%$ & 10 & $26,3 \%$ \\
\hline de 60 a 69 anos & 5 & $13,2 \%$ & 5 & $13,2 \%$ & 10 & $26,3 \%$ \\
\hline de 70 a 80 anos & 9 & $23,7 \%$ & 9 & $23,7 \%$ & 18 & $47,4 \%$ \\
\hline Total & 19 & $50,0 \%$ & 19 & $50,0 \%$ & 38 & $100 \%$ \\
\hline \multicolumn{7}{|l|}{ Masculino } \\
\hline Grupo & \multicolumn{2}{|c|}{ Não parkinsoniano } & \multicolumn{2}{|c|}{ Parkinsoniano } & \multicolumn{2}{|c|}{ Geral } \\
\hline Faixa Etária & Absoluto & Relativo & Absoluto & Relativo & Absoluto & Relativo \\
\hline de 50 a 59 anos & 6 & $14,3 \%$ & 6 & $14,3 \%$ & 12 & $28,6 \%$ \\
\hline de 60 a 69 anos & 8 & $19,0 \%$ & 8 & $19,0 \%$ & 16 & $38,1 \%$ \\
\hline de 70 a 80 anos & 7 & $16,7 \%$ & 7 & $16,7 \%$ & 14 & $33,3 \%$ \\
\hline Total & 21 & $50,0 \%$ & 21 & $50,0 \%$ & 42 & $100 \%$ \\
\hline
\end{tabular}




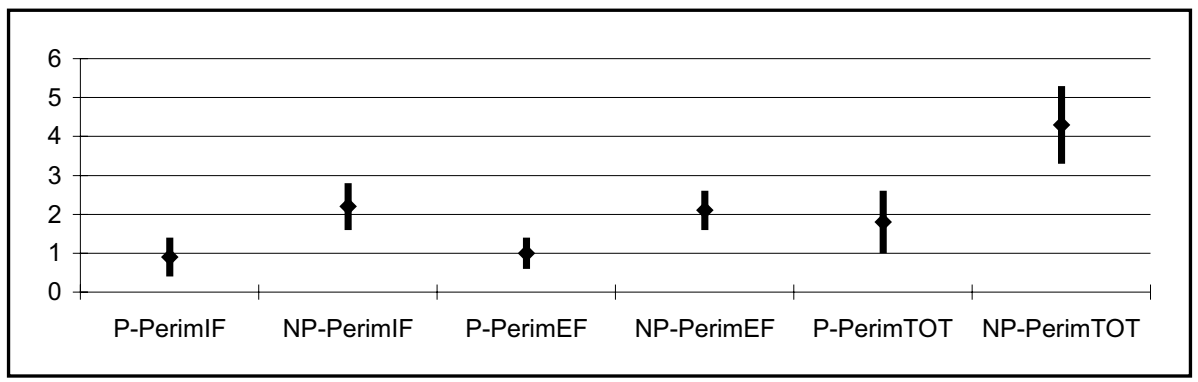

Fig 1. Médias e desvios-padrão dos resultados da perimetria inspiratória (PerimIF), da perimetria expiratória (PerimEF) e da perimetria total (PerimTOT) nos grupos parkinsonianos $(P)$ e não parkinsonianos (NP).

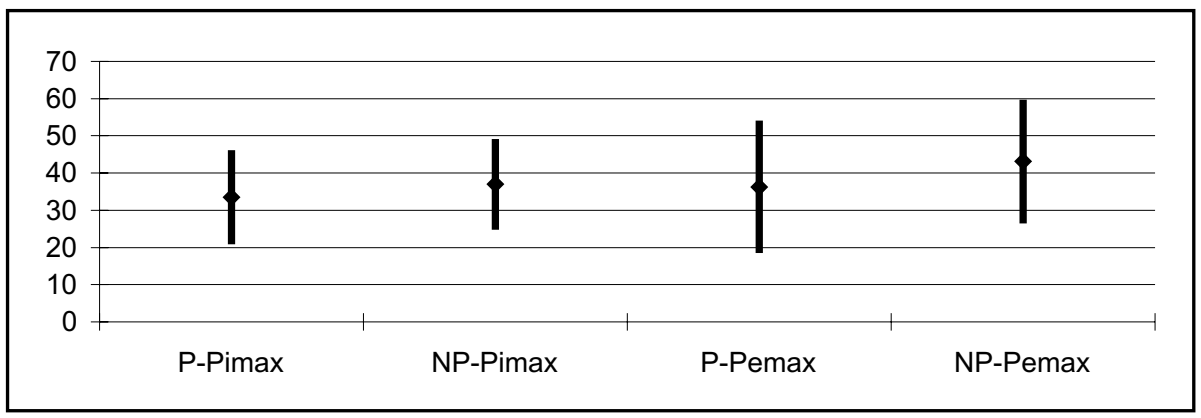

Fig 2. Médias e desvios-padrão dos resultados da pressão inspiratória máxima (Pımax) e da pressão expiratória máxima (Pemax) nos grupos parkinsonianos ( $P$ ) e não parkinsonianos (NP).

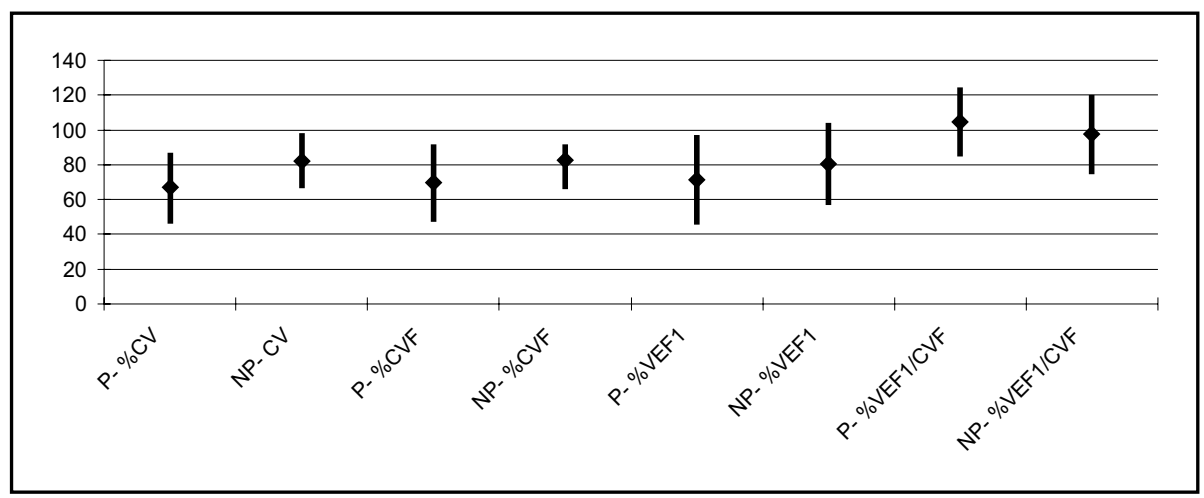

Fig 3. Médias dos resultados e desvios-padrão da capacidade vital em relação aos valores teóricos $(\% C V)$, da capacidade vital forçada em relação aos valores teóricos (\%CVF), do percentual de volume do primeiro segundo da curva expiratória forçada em relação aos valores teóricos (\%VEF $)$, e do percentual da capacidade vital forçada expirada em um segundo, em relação aos valores teóricos (\%VEF, /CVF) nos grupos parkinsonianos ( $P$ ) e não parkinsonianos (NP).

relação aos valores teóricos (\%CVF), de 69,6 $22,2 \%$ nos $\mathrm{P}$ e $82,7 \pm 16,6 \%$ nos NP, também se apresentou significativamente menor $(p=0,0023)$ nos $P$. O percentual de volume do primeiro segundo da curva expiratória forçada em relação aos valores teóricos $\left(\% \mathrm{VEF}_{1}\right)$, de $71,3 \pm 25,6 \%$ nos $\mathrm{P}$ e $80,6 \pm 23,6 \%$ nos NP e o percentual da capacidade vital forçada expirada em um segundo, em relação aos valores teóricos (\% $\left.\mathrm{VEF}_{1} / \mathrm{CVF}\right)$, de $104,5 \pm 19,9 \%$ nos $\mathrm{P}$ e $97,4 \pm 22,8 \%$ nos $N P$, não evidenciaram diferença significativa (Fig 3).

No resultado espirométrico dos $P$ foram mais evidentes os quadros que envolvem restrição (70\%), seguindo-se o normal (25\%) e os que envolvem obstrução $(2,5 \%)$ equiparados aos que associam restrição e obstrução (2,5\%). Nos NP o normal ocorreu na maioria (55\%), seguido dos que envolvem obstrução $(10 \%)$, restrição (25\%) e dos que associam restrição e obstrução (10\%) (Tabela 2).

As relações entre Pimax e amplTOT, definida pela sua razão, resultou em $19,8 \pm 8,2$ nos $P$ e $8,7 \pm 2,3$ nos NP (Tabela 3) e Pemax e amplTOT, em 20,3 $\pm 6,2$ nos $P$ e $10,1 \pm 3,3$ nos NP, identificando diminuição significativa entre as médias da variável, nos NP ( $p$ $=0,00001$ ) (Tabela 4). 
Tabela 2. Resultado espirométrico.

\begin{tabular}{lcccccc}
\hline $\begin{array}{l}\text { Grupo } \\
\text { Resultado Espirometria }\end{array}$ & \multicolumn{2}{c}{ Não parkinsonismo } & \multicolumn{2}{c}{ Parkinsonismo } & \multicolumn{2}{c}{ Geral } \\
\hline Normal & Absoluto & Relativo & Absoluto & Relativo & Absoluto & Relativo \\
Obstrutivo leve & 22 & $27,5 \%$ & 10 & $12,5 \%$ & 32 & $40,0 \%$ \\
Obstrutivo moderado & 2 & $2,5 \%$ & 1 & $1,3 \%$ & 3 & $3,8 \%$ \\
Restritivo leve & 2 & $2,5 \%$ & 0 & $0,0 \%$ & 2 & $2,5 \%$ \\
Restritivo leve associado & 6 & $7,5 \%$ & 16 & $20,0 \%$ & 22 & $27,5 \%$ \\
$\quad$ a obstrutivo leve & 2 & $2,5 \%$ & 0 & $0,0 \%$ & 2 & $2,5 \%$ \\
Restritivo leve associado & & & & & \\
$\quad$ a obstrutivo moderado & 2 & $2,5 \%$ & 1 & $1,3 \%$ & 3 & $3,8 \%$ \\
Restritivo moderado & 4 & $5,0 \%$ & 6 & $7,5 \%$ & 10 & $12,5 \%$ \\
Restritivo severo & 0 & $0,0 \%$ & 6 & $7,5 \%$ & 6 & $7,5 \%$ \\
Total & 40 & $50,0 \%$ & 40 & $50,0 \%$ & 80 & $100 \%$ \\
\hline
\end{tabular}

Tabela 3. Análise da relação pressão inspiratória máxima/amplitude total.

\begin{tabular}{lccl}
\hline & $\mathrm{F}$ & $\mathrm{p}$ & Tukey \\
\hline Geral & 19,178 & 0,00001 & $\begin{array}{l}\text { Existe Diferença } \\
\text { Não parkinsonianos } \\
\text { Grupo }\end{array}$ \\
$\begin{array}{lccl}\text { < Parkinsoniano } \\
\text { Sexo }\end{array}$ & 0,49064 & 0,4897 & Iguais \\
Faixa Etária & 2,669 & 0,0763 & Iguais \\
2 - Interações & 1,605 & 0,1701 & Iguais \\
Grupo Vs. Sexo & 3,120 & 0,0817 & Iguais \\
Grupo Vs. Faixa Etária & 1,632 & 0,2030 & Iguais \\
Sexo Vs Faixa Etária & 0,989 & 0,3771 & Iguais \\
\hline
\end{tabular}

Tabela 4. Análise da relação pressão expiratória máxima/amplitude total.

\begin{tabular}{lccl}
\hline & $\mathrm{F}$ & $\mathrm{p}$ & Tukey \\
\hline Geral & 23,743 & 0,00001 & $\begin{array}{l}\text { Existe Diferença } \\
\text { Não parkinsonianos } \\
\text { Grupo }\end{array}$ \\
$\begin{array}{lccl}\text { < Parkinsoniano } \\
\text { Sexo }\end{array}$ & 2,044 & 0,00001 & Iguais \\
Faixa Etária & 1,973 & 0,0926 & Iguais \\
2 - Interações & 0,957 & 0,4506 & Iguais \\
Grupo Vs. Sexo & 0,533 & 0,4756 & Iguais \\
Grupo Vs. Faixa Etária & 1,597 & 0,2097 & Iguais \\
Sexo Vs. Faixa Etária & 0,480 & 0,6208 & Iguais \\
\hline
\end{tabular}

\section{DISCUSSÃO}

As medidas da PerimlF, PerimEF e PerimTOT foram significativamente menores nos parkinsonianos, caracterizando acentuada diminuição da sua mobilidade torácica durante a respiração e repercutindo no aumento do trabalho muscular, com conseqüente diminuição da expansibilidade pulmonar na inspiração e da depressão torácica na expiração. Comprovou-se que o tórax rígido e resistente aos movimentos rápidos acarreta limitação progressiva da ventilação ${ }^{8}$, a fixação postural em flexão e a rigidez da musculatura intercostal comprometem a mobili- dade da caixa torácica ${ }^{5,9}$ e a artrose e a cifoescoliose reduzem a mobilidade da coluna e do tórax, sendo a bradicinesia um fator interferencial ${ }^{3,6}$.

Em nosso estudo, as medidas da Pimax e da Pemax não evidenciaram diferença entre $P$ e NP, enquanto Canning e col. ${ }^{10}$ identificaram diminuição da PImax em apenas um dos 60 parkinsonianos examinados, entre 47 e 60 anos, classificados nos níveis IIII da H-Y, embora outros autores encontrassem esta redução em um número maior de indivíduos ${ }^{4,11}$. Medidas na Pimax de $-33,17 \mathrm{cmH}_{2} \mathrm{O}$ foram observadas em 10 parkinsonianos com $51 \pm 5,3$ anos, des- 
tes, cinco encontravam-se na fase "on", entre os níveis I-III da $\mathrm{H}-\mathrm{Y}^{12}$. Sabaté e col. ${ }^{3}$ encontraram valores menores, em torno de $-25,1 \pm 17,2 \mathrm{cmH}_{2} \mathrm{O}$, observando-se no grupo controle valores de 52,1 $\pm 24,2$ $\mathrm{cmH}_{2} \mathrm{O}$. Alguns autores ${ }^{4,13}$ associaram esta redução a fadiga muscular, embora outros ${ }^{14}$ admitissem sua relação com a gravidade do quadro neurológico.

Existem discordâncias na literatura em relação a Pemax dos parkinsonianos, não ocorrendo alterações ${ }^{10}$ como em nosso estudo ou observando-se redução da mesma ${ }^{11}$, o que pode estar relacionado com o comprometimento neurológico desses pacientes $^{14}$. Enquanto De Bruin e col. ${ }^{12}$ registraram Pemax de $63 \pm 29 \mathrm{cmH}_{2} \mathrm{O}$ em indivíduos parkinsonianos, com $51 \pm 5,3$ anos de idade, Sabaté e col. ${ }^{3}$ obtiveram valores menores, em torno de $38,4 \pm 13,3 \mathrm{cmH}_{2} \mathrm{O}$, em indivíduos mais idosos, com $67,7 \pm 1,06$ anos, registrando-se medidas de $63,5 \pm 23,28 \mathrm{cmH}_{2} \mathrm{O}$ para o grupo controle. Ressalta-se na expiração: a diminuição da força muscular expiratória, a dificuldade de contração muscular rápida e a atividade constante da musculatura intercostal ${ }^{4}$.

As medidas da capacidade vital e da capacidade vital forçada, em relação aos valores teóricos $(\% \mathrm{CV}$ e \%CVF), significativamente menores nos parkinsonianos, evidenciaram a diminuição da expansibilidade pulmonar, classificando-a, através da espirometria, como um quadro pulmonar restritivo característico na DP, como constatado por O'Sullivan \& Schmitz $^{5}$. Estudos analisando a função respiratória em 63 parkinsonianos com 67,1 $\pm 0,96$ anos evidenciaram diminuição da complacência pulmonar e presença de microatelectasias em $85 \%^{2}$. Observou-se, ainda, limitação na extensão do tronco, na amplitude articular e na curvatura patológica na coluna vertebral, relacionando-se a CVF com a bradicinesia e a rigidez torácica, com a obtenção de valores de $81,3 \pm$ $22,3 \%$, medidos em 58 parkinsonianos de $67,7 \pm 1,06$ anos $^{6}$. De Bruin e col. ${ }^{12}$ encontraram valores de 86,5 $\pm 23,6 \%$ para a CVF, associadas a alterações da musculatura torácica, podendo esta restrição ser devido à fibrose pleural ${ }^{4}$.

Análise dos percentuais de volume da curva expiratória forçada (\%VEF ) e da capacidade vital forçada que pode ser expirada em um segundo $\left(\% \mathrm{VEF}_{1} /\right.$ CVF) como referenciais da permeabilidade de vias aéreas, quando relacionados entre si, não caracterizaram a presença de obstrução brônquica nos parkinsonianos ${ }^{13-15}$. O VEF 1 foi normal na doença relativamente severa ${ }^{10,11}$, com medidas de $88,7 \pm 23,8 \%{ }^{6}$ até $108,1 \pm 3,4 \%{ }^{2}$. Um estudo utilizando 10 parkinsonianos, entre 42 e 60 anos, observou apenas um deles com padrão obstrutivo ${ }^{12}$; entretanto outro estudo evidenciou cerca de $56 \%$ de parkinsonianos obstrutivos dos $84 \%$ que registraram alterações espirométricas, relacionando estes resultados com a presença de bradicinesia no movimento respiratório e postura patológica crônica ${ }^{3}$.

Concluímos que a diminuição da amplitude torácica foi o fator determinante das alterações respiratórias restritivas dos parkinsonianos, limitando a elevação das estruturas do tórax e a expansibilidade pulmonar. A presença de obstrução brônquica não foi significativa e a força muscular respiratória mostrou-se equivalente entre os grupos estudados, embora a depressão torácica tenha apresentado também limitação por resistência imposta à mobilização torácica pela postura em flexão e pela presença de rigidez, típicas da DP. Desta forma, há necessidade de um programa de tratamento preventivo ou reabilitador, direcionado para o aumento da amplitude torácica, o que promoverá a melhora da função respiratória e da capacidade funcional desses pacientes, trazendo-lhes maior independência e, por conseguinte, melhor qualidade de vida.

\section{REFERÊNCIAS}

1. Köseoglu F, Inan L, Ozel S et al. The effects of a pulmonary rehabilitation program on pulmonary function tests and exercise tolerance in patients with Parkinson's disease. Funct Neurol 1997;12:319-325.

2. Izquierdo-Alonso JL, Jimenez-Jimenez FJ, Cabrera-Valdivia F, MansillaLesmes M. Airway dysfunction in patiernts with Parkinson's disease. Lung 1994;172:47-55.

3. Sabaté M, Rodríguez M, Mendez E, Enríquez E, González I. Obstructive and restrictive pulmonary dysfunction increases disability in Parkinson disease. Arch Phys Med Rehabil 1996b;77:29-34.

4. Brown LK. Respiratory dysfunction in Parkinson's disease. Clin Chest Med 1994;15:715-727.

5. O'Sullivan SB, Schmitz TJ. Fisioterapia: avaliação e tratamento. São Paulo: Manole 1993:549-564.

6. Sabaté M, González I, Ruperez F, Rodríguez M. Obstructive and restrictive pulmonary dysfunctions in Parkinson's disease. J Neurol Sci 1996a;138:114-119.

7. Brandstetter RD, Kazemi, H. Envelhecimento do sistema respiratório. Clínicas Médicas da América do Norte. Rio de Janeiro: Interamericana 1983;2:441-453.

8. MacIntosh DJ. Respiratory dysfunction in Parkinson disease. Prim Care 1977;4:441-445.

9. Limongi JCP. Doença de Parkinson. Rev Bras Med 1993;50:1079-1084.

10. Canning CG, Allison JA, Allen NE, Groeller H. Parkinson's disease: an investigation of exercice capacity, respiratory function, and gait. Arch Phys Med Rehabil 1997;78:199-207.

11. Hovestadt A, Bogaard JM, Meerwaldt JD, van der Meche FG, Stigt J. Pulmonary function in Parkinson's disease. J Neurol Neurosurg Psychiatry 1989;52:329-333.

12. De Bruin, PFC, De Bruin, VMS, Lees AJ, Pride, NB. Effects of treatment on airway dynamics and respiratory muscle strength in Parkinson's disease. Annu Rev Respir Dis 1993;148:1576-1580.

13. Tzelepis GE, McCool FD, Friedman JH, Hoppin FGJr. Respiratory muscle dysfunction in Parkinson's disease. Annu Rev Respir Dis 1988; 138:266-271

14. Bogaard JM, Hovestadt A, Meerwaldt JVD, van der Meche FG, Stigt J. Maximal expiratory and inspiratory flow-volume curves in Parkinson's disease. Annu Rev Respir Dis 1989;139:610-614.

15. Murdoch BE, Chenery HJ, Bowler S, Ingram JC. Respiratory function in Parkinson's subjects exhibiting a perceptible deficit: a kinematic and spirometric analysis. Chest 1989;96:210-212. 